\title{
Secured QR-Code based COD payment using Mobile Bill Presentment System
}

\author{
Harish C. V. ${ }^{1}$, Dr. Ravishankar. $S^{2}$ \\ ${ }^{l} C M R T U, R$ V College Campus, ${ }^{2}$ Department of ECE, $R$ V College of Engineering \\ Email: kbondharish@gmail.com ${ }^{1}$, ravishankars@rvce.edu.in ${ }^{2}$
}

\begin{abstract}
Worldwide Cash on Delivery (COD) and Point of Sale (POS) is the online shopping payment systems. Some of the limitations in cash on delivery and point of sale are the risk in handling the hard cash by the delivery person, chances of getting fake notes, the service charges which has to be paid by the merchant for every transactions of using POS machine which is a burden to the merchant. To overcome these limitations, a new technology known as mobile bill presentment system (MBPS) is introduced which uses present most useful hardware by every person in the world. To make online payments, security is the major aspect to develop any system for payment. The POS is maintaining the security but the above limitations made this work to be carried out. The Quick Response (QR) Code is developed and installed in the MBPS hardware system and payment for the online purchased product is made easy and more secured for both merchant and the purchaser. The most useful hardware is the smart phones which is used and have the connectivity to make all payments easily through smart phones.
\end{abstract}

Keywords: Cash on Delivery (COD), Point of Sale (POS), Mobile Bill Presentment System (MBPS), Quick Response (QR) Code

\section{INTRODUCTION}

Mobile Bill presentment System is a Mobile Application which enables the user to pay bill by scanning the QR code present in the Bill. The mobile acts as an intermediate which scans the QR code and takes the user to the payment gateway and enables the user to pay the bill without much difficulty.

It is a unique and new product present in the current market scenario. The bill has a unique $\mathrm{QR}$ code present in it, which could be only read using the Mobile application and comes with a secure mode of payment.

This is an electronic version of the traditional bill payment system which would help our customers to make the payment of their bills by scanning the Quick Response (QR) Code of the bill through a Smartphone.

The COD is one of the widely used and is simple methodology for the purchaser to make the payment for online shopping cart. The customer place the order, the merchant provides the facility of homme delivery to the customer to avail their ordered product. The customer has to pay the amount of their product only at the time of delivery of the product. The main feature of COD attracts the customer to make the payment once the product is delivered, the customer need not worry about the ordered product and need not track the destination of the product since the product is not lost and if it is lost need not payed. Based on these features, the COD is the most widely used method to make payments for online shopping applications worldwide.

The other method of making the payment is point of sale (POS). The point of sale is the hardware device which make use of debit or credit card swiping for payments. This system of payment requires the magnetic card. The system consists of magnetic tape which reads the card information and connect to the network through GPRS and get the authentication from the card holder and confirm the payment and receipt is printed on the system. This system is also most widely used payment system. The major limitations of the system are, the merchant has to pay the service charges for the POS system, the delivery person has to carry the machine every where, and always network connectivity is required [1][2].

The proposed work is to overcome the limitations of the COD and POS payment system. Second section of this paper gives the methodology followed to carry out this work. Third section consists of algorithm used to implement and outputs. The foruth section is results and conclusions.

\section{METHODOLOGY}

To overcome the above mentioned limitations of the existing payment system an innovative application is introduced to make a hassle free payment and is known as Mobile Bill Presentment system (MBPS). Fig. 1 shows the block diagram of the MBPS system.

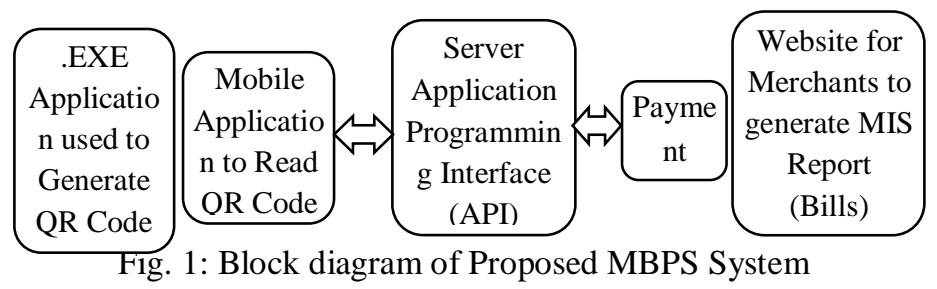

This application contains four modules: 1. An EXE application which has to be integrated on the merchant software to generate QR bills. 2. An android Mobile application to read this QR image and make payment. 3. Server Application Programming Interface (API). 4. Redirecting to payment gateway and payment acknowledgment. 5. A website for merchants to generate various MIS Reports.

Android is a mobile operating system (OS) currently developed by Google, based on the Linux kernel and designed primarily for touchscreen mobile devices such 
as smartphones and tablets [3]. Android's user interface is mainly based on direct manipulation, using touch gestures that loosely correspond to real-world actions, such as swiping, tapping and pinching, to manipulate on-screen objects, along with a virtual keyboard for text input.

- This smart cart is a software application embedded with hardware and broadcasting network layers.

- The engine recognizes the price and configuration of the related products and helps the user to compare between each product.

- This system is been developed to reduce the chances of the user losing their money and to reduce the fraudulent.

- Through this system payment options could be made more secure and safe to do the transactions. Multilevel encryption is applied in different layer of projects also.

- The process of payment involved submission of cash by the customer and an issuance of acknowledgement (paper payment receipt) by the merchant.

- Mobile Bill presentment System is a Smart Mobile Phone (an electronic device) Application which enables the user to pay bill by scanning the QR code present in the Bill using mobile camera.

- The mobile acts as an intermediate which scans the QR code and takes the user to the payment gateway and enables the user to pay the bill without much difficulty.

- It is a unique and new product present in the current market scenario. The bill has a unique QR code present in it, which could be only read using the Mobile application and comes with a secure mode of payment.

- This is an electronic version of the traditional bill payment system which would help our customers to make the payment of their bills by scanning the Quick Response (QR) Code of the bill through a Smartphone.

Fig. 2 shows the high level architecture of the complete system on which the proposed system works. The QR code will be generated when the end user purchase an article, the generated QR code will be stored in the server. When delivery person comes and ask for the payment, simply scan the QR code shown by the delivery person, verify the product and amount to be paid. The end user can pay the amount by selecting one of the options using different payment gateway.

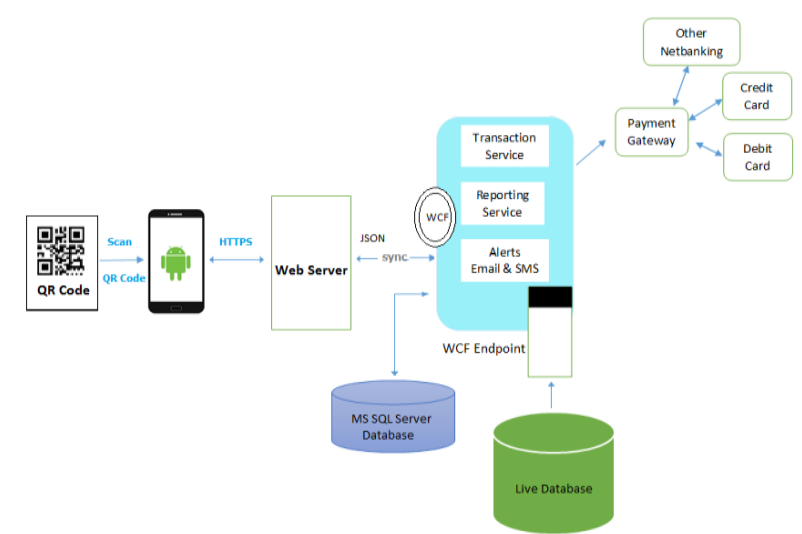

Fig. 2: High Level Architecture of MBPS

During the process of scanning QR code and payment method, the hardware used are smartphone, web server, WCF endpoint, payment gateway. Smartphone is installed with MBPS application software. Smartphone scans the QR code and send it to web server through HTTP protocol. The web server sends it to WCF endpoint. WCF endpoint verifies the $\mathrm{QR}$ code by fetching the stored information from live database. Once the verification is done and it is valid then the smartphone will get payment page and proceed for the payment. Payment process takes place through payment gateway through WCF endpoint. The payment options are Net banking, Credit or Debit card is selected in smartphone using the input device.

All communication with a Windows Communication Foundation (WCF) [4] service occurs through the endpoints of the service. Endpoints provide clients access to the functionality offered by a WCF service.

JSON [5][6][7] is a minimal, readable format for structuring data. It is used primarily to transmit data between a server and web application, as an alternative to XML [8]. JSON is a format that encodes objects in a string. Serialization means to convert an object into that string, and deserialization is its inverse operation. When transmitting data or storing them in a file, the data are required to be byte strings, but complex objects are seldom in this format.

Request initiating from smartphone $\rightarrow$ Server receives the request and process $->$ server is using database to store the details $\rightarrow$ after processing the details, server will give response to smartphone $\rightarrow$ smartphone reads the response and display appropriate message.

\section{ALGORITHM AND OUTPUT}

\author{
SERVICE: TRANSACTION \\ INPUT \\ \{ \\ "BillerName": "MERCHANT NAME", \\ "CustomerID": "10240522", \\ "MobileNumber": "9986829509", \\ "BillNumber": "352162566682", \\ "BillPeriod": "01.04.2014 to 30.04.2014", \\ "TotalAmount": "9200.50", \\ "Channel": "G"
}




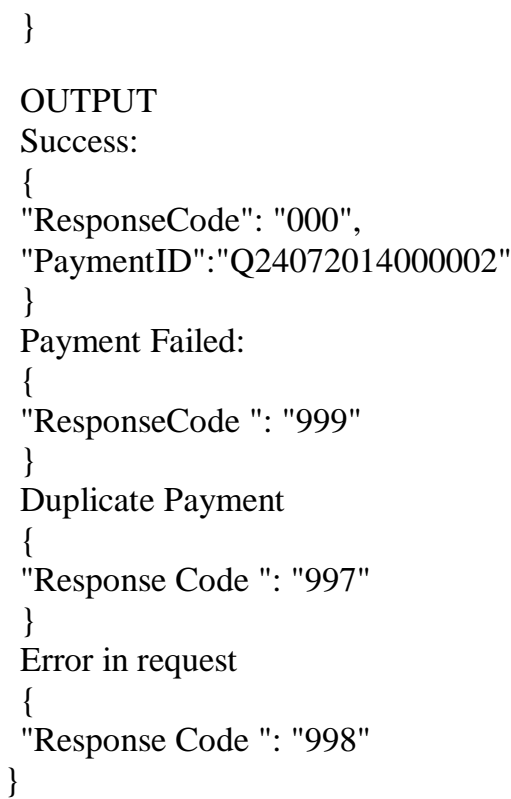

The description of each key value pair is detailed below. Input parameters:

- The operation requested is Transaction to mobile application.

- Biller Name - Name of the Merchant.

- Customer ID - ID of the Customer registered with Merchant.

- Mobile Number - Mobile number of the Customer registered with Merchant.

- Bill Number -ID of Customer's bill.

- Bill Period - Billing Period of the Customer.

- Total Amount - Total Amount of Customer's bill.

- Channel -Payment Channel selected by the Customer to pay the bill i.e. Either V (VNetBanking) or G (Payment Gateway)

All data representation is an encrypted Hash value of the Payment Channel selected by the Customer for making payment.

Output Parameters:

\section{For success case:}

Response code: 000 - Response code value to indicate that operation performed is success.

Payment ID - Payment ID of the Transaction

For failure case of transaction:

Response code: 999 - Transaction has failed.

For duplicate payment:

Response Code: 997- The bill has already been paid by the customer.

\section{For Error in Request:}

Response Code: 998 - Invalid parameters in the payment request.

\section{RESULTS AND CONCLUSIONS}

A new innovative dimension of all types of bill payment has been invented here which is named as Mobile Bill Presentment System (MBPS). Using various technology, we have evolved with a very useful and compact way of bill payment which is highly convenient for the customer and economically feasible for merchants.

This innovative product overcomes all the limitations of the present bill payment scenario. MBPS is completely travelling on a secured electronic channels which does not involve any sort of paper receipts. This product is highly useful for the customer and the merchant as it is user friendly and economic. MBPS is also an ecofriendly product as it completely avoids all kind of paper wastage because no hard cash and no paper receipts are provided in MBPS which will give us a greener environment.

\section{REFERENCES}

[1] The Benefits and Risks of Cloud Point-of-Sale via Tapas Technologies

[2] Jump up, Frazier, Bob (April 19, 2014). "Restaurant POS Software List". POS Advice Link.

[3] Jump up Micro-payment systems and their application to mobile networks, InfoDev report

[4] Large-Scale Automated Identification and Quality Control of Exfoliated and CVD Graphene via Image Processing Technique, C. M. Nolena, D. Teweldebrhana , G. Deninab , B. Bhanub , and A. A. Balandina

[5] A. Sankara Narayanan, " $Q R$ Codes and Security Solutions", International Journal of Computer Science and Telecommunications [Volume 3, Issue 7, July 2012].

[6] Jun-Chou Chuang, Yu-Chen $\mathrm{Hu} \&$ Hsien-Ju Ko, " $A$ Novel Secret Sharing Technique Using QR Code", International Journal of Image Processing (IJIP), Volume (4) : Issue (5).

[7] Design and Implementation of Rijndael Encryption Algorithm Based on FPGA, K. Soumya1 , G. Shyam Kishore.

[8] SQUARESPACE: http://developers.squarespace.com/what-is-json/ 